\title{
Clinical PET-CT in Radiology: Integrated Imaging in Oncology
}

P. Shreve and D.W. Townsend, eds.

New York, NY: Springer, 2011, 437 pages, $\$ 189$

Hybrid imaging and diagnosis by multimodality imaging are broad and pervasive topics in the medical imaging field. The practice of integrating the interpretation of complementary imaging modalities for clinical diagnosis has been ongoing in disease-based and organ system-based medical subspecialties. This trend has recently accelerated with the widespread application of computer technologies and continued refinements of image-registration and -fusion software. The remarkably fast adoption of PET/CT in the last decade was due to its ability to provide morphologic as well as metabolic information, leading to overwhelming clinical applications in oncology.

This comprehensive textbook presents the latest hybrid PET/CT technology for applications in cancer management, particularly body areas for which correlation with other radiologic imaging is available. The editors have brought together an internationally distinguished group of 50 authors to cover all aspects of clinical PET/CT relevant to body oncologic procedures.

The 32 chapters cover integrated morphologic-metabolic imaging as applied to cancer imaging, with a concise and practical guide for the practicing imaging physician. Chapters 1-13 deal with instruments, basic imaging sciences, and artifacts of PET/CT, as well as patient preparation, radiation protection, and imaging interpretation. Chapters 14 and 15 discuss principles of medical imaging, as well as PET in cancer staging and treatment. Chapters 16-32 are clinical chapters arranged by organ system, and each chapter helps the imaging physician understand the relationship and role of the integrated imaging modality with respect to the overall management of cancer patients. The 303 figures are clear, the 140 color images illustrative, the 81 tables informative, and the references updated.

This book provides a concise and structured background on the management of cancer and the role of PET/CT, which is in the mainstream of oncologic imaging. I am sure that this book will be a valuable companion for imaging physicians and will also be helpful for oncologists, general physicians, and imaging trainees.

\section{E. Edmund Kim}

M.D. Anderson Cancer Center

1515 Holcombe Blvd., Unit 1264

Houston, TX 77030

E-mail:ekim@mdanderson.org

COPYRIGHT (C) 2012 by the Society of Nuclear Medicine, Inc.

Published online Jan. 25, 2012.

DOI: 10.2967/jnumed.111.097535 